\title{
Modulation of Cerebellar Excitability by Polarity-Specific Noninvasive Direct Current Stimulation
}

\author{
Joseph M. Galea, ${ }^{1}$ Gowri Jayaram, ${ }^{2}$ Loni Ajagbe, ${ }^{1}$ and Pablo Celnik ${ }^{1}$ \\ ${ }^{1}$ Department of Physical Medicine and Rehabilitation, Johns Hopkins Medical Institution, Baltimore, Maryland 21231, and ${ }^{2}$ Department of Biomedical \\ Engineering, School of Medicine, Johns Hopkins University, Baltimore, Maryland 21205
}

\begin{abstract}
The cerebellum is a crucial structure involved in movement control and cognitive processing. Noninvasive stimulation of the cerebellum results in neurophysiological and behavioral changes, an effect that has been attributed to modulation of cerebello- brain connectivity. At rest, the cerebellum exerts an overall inhibitory tone over the primary motor cortex (M1), cerebello- brain inhibition (CBI), likely through dentate-thalamo- cortical connections. The level of excitability of this pathway before and after stimulation of the cerebellum, however, has not been directly investigated. In this study, we used transcranial magnetic stimulation to determine changes in M1, brainstem, and CBI before and after $25 \mathrm{~min}$ of anodal, cathodal, or sham transcranial direct current stimulation (tDCS) applied over the right cerebellar cortex. We hypothesized that anodal tDCS would result in an enhancement of CBI and cathodal would decrease it, relative to sham stimulation. We found that cathodal tDCS resulted in a clear decrease of CBI, whereas anodal tDCS increased it, in the absence of changes after sham stimulation. These effects were specific to the cerebello-cortical connections with no changes in other M1 or brainstem excitability measures. The cathodal effect on CBI was found to be dependent on stimulation intensity and lasted up to $30 \mathrm{~min}$ after the cessation of tDCS. These results suggest that $\mathrm{tDCS}$ can modulate in a focal and polarity-specific manner cerebellar excitability, likely through changes in Purkinje cell activity. Therefore, direct current stimulation of the cerebellum may have significant potential implications for patients with cerebellar dysfunction as well as to motor control studies.
\end{abstract}

\section{Introduction}

The cerebellum plays an important role in the planning, initiation, stability, organization, and long-term memory of movements (Imamizu et al., 2000; Chen et al., 2006; Morton and Bastian, 2006; Miall et al., 2007). Therefore, developing strategies to modulate cerebellar excitability is of significant interest to further the understanding of its function and as a potential rehabilitation strategy for patients with cerebellar diseases.

Previous studies aiming to modulate cerebellar excitability have applied slow repetitive transcranial magnetic stimulation (rTMS) over the cerebellum and determined the neurophysiological effects indirectly on the primary motor cortex (M1) (Oliveri et al., 2005; Fierro et al., 2007; Koch et al., 2008; Langguth et al., 2008). However, the findings have been inconsistent, where some described an increase in intracortical M1 excitability (Oliveri et al., 2005; Koch et al., 2008) and others the opposite (Fierro et al., 2007; Langguth et al., 2008). Other investigations have reported the behavioral effects of cerebellar rTMS such as increased variability of finger tapping (Theoret et al., 2001) and reduced essential tremor (Gironell et al., 2002). Only one study has used transcranial direct current stimulation (tDCS), a noninvasive, painless form of stimulation that can enhance (anodal)

\footnotetext{
Received May 8, 2009; revised June 8, 2009; accepted June 16, 2009.

This work was supported by National Institute of Child Health and Human Development-National Institutes of Health Grants R01 HD053793 and R21 HD060169.

Correspondence should be addressed to Pablo Celnik, Department of Physical Medicine and Rehabilitation, Johns

Hopkins Medical Institution, 98 North Broadway, Baltimore, MD 21231. E-mail: pcelnik@jhmi.edu.

D0I:10.1523/JNEUROSCI.2184-09.2009

Copyright $\odot 2009$ Society for Neuroscience $\quad 0270-6474 / 09 / 299115-08 \$ 15.00 / 0$
}

or decrease (cathodal) excitability in a polarity-specific manner (Nitsche and Paulus, 2000, 2001), over the cerebellum and showed that both anodal and cathodal tDCS impaired the practice-dependent proficiency increase in working memory (Ferrucci et al., 2008). These studies suggested that the mechanism responsible for the physiological or behavioral effect involved modulation of the cerebellar output to other brain structures. However, the exact mechanism was not directly assessed, an important issue to be elucidated to be able to guide in a rational manner interventions that can modulate cerebellar excitability.

One of the main outputs of the cerebellum is the dentatethalamo-cortical pathway. The Purkinje cells, the output neurons of the cerebellar cortex, have inhibitory connections with the dentate cerebellar nucleus (DCN), which in turn has a disynaptic excitatory connection through the thalamus to M1 (Middleton and Strick, 2000; Kelly and Strick, 2003). Thus, Purkinje cell activity exerts an inhibitory tone over M1, referred to as cerebello-brain inhibition (CBI). Evidence for the presence of $\mathrm{CBI}$ comes from studies evaluating motor cortical excitability in healthy individuals and patients with cerebellar stroke or degeneration. The latter have consistently shown enhancement of corticomotor inhibition and reduction in motor facilitation secondary to lesions of the DCN (Liepert et al., 2004; Battaglia et al., 2006). In healthy humans, the dentate-thalamo-cortical pathway has been assessed noninvasively through electrical and magnetic stimulation of the cerebellum (Ugawa et al., 1991, 1995; Pinto and Chen, 2001; Daskalakis et al., 2004). Therefore, it would be possible to directly determine the effect of cerebellar stimulation by evaluating changes in $\mathrm{CBI}$. 
In this study, we aimed to explore the effects of noninvasive cerebellar stimulation in healthy individuals by assessing changes in excitability in M1, brainstem, and cerebellum before and after application of cerebellar anodal, cathodal, or sham tDCS. We hypothesized that anodal stimulation would increase CBI and cathodal would decrease it relative to sham.

\section{Materials and Methods \\ Subjects}

Sixteen right-handed healthy individuals with no history of neurological or psychiatric conditions (six women; mean age, $26 \pm 7$ years; range, $20-45$ years) participated in the study. All subjects signed informed consent approved by the Johns Hopkins Medical Institution Institutional Review Board and in accordance with the Declaration of Helsinki.

\section{Experiment 1: modulation of cerebellar excitability by $t D C S$}

Eight subjects (four women; mean age, $28 \pm 10$ years; range, 20-45 years) participated in a crossover study, which consisted of three randomized ordered sessions, separated by at least $6 \mathrm{~d}$ (Fig. 1). The order of physiological assessments before and after the application of cerebellar tDCS remained consistent across sessions. At the end of each session, subjects reported their attention, fatigue, and perceived pain of tDCS using a self-scored visual analog scale in which 1 represented poorest attention, maximal fatigue, and pain and 7 represented maximal attention, least fatigue, and pain (see Table 2) (Stefan et al., 2005).

\section{Experimental procedures}

EMG recordings. Subjects sat comfortably in a chair with both arms resting on a pillow placed on their lap. Electromyographic (EMG) activity was captured through pairs of disposable electrodes placed over the right first dorsal interosseus (FDI) and the left and right pectoralis major muscles. EMG signals were recorded, amplified, and filtered using a Viking IVP (Nicolet; bandwidth, $5 \mathrm{~Hz}$ to $1 \mathrm{kHz}$; Viasys Healthcare) and sampled at $1 \mathrm{kHz}$ through Signal software (version 4.02; CED). The data were stored on a personal computer for off-line analysis using a custom Matlab program (MathWorks).

tDCS. tDCS was delivered through two sponge electrodes (surface area, $25 \mathrm{~cm}^{2}$ ) embedded in a saline-soaked solution. One electrode was centered on the right cerebellar cortex, $3 \mathrm{~cm}$ lateral to the inion (Ugawa et al., 1995). The other electrode was positioned on the right buccinator muscle (Fig. 1b). This is in contrast to Ferrucci et al. (2008), who positioned the electrode on the deltoid muscle. We avoided that location because of the concern of inducing excitability changes in the brachial plexus and thus affecting our measures obtained from arm and trunk muscles. At the onset of all interventions (anodal, cathodal, and sham), current was increased in a ramp-like manner as done in previous reports (Nitsche et al., 2003a; Hummel et al., 2005). The intensity of stimulation was set at $2 \mathrm{~mA}$ (Iyer et al., 2005; Ferrucci et al., 2008), and unbeknown to the subject, anodal or cathodal tDCS was delivered over the cerebellum for 25 min using a Phoresor II Auto (model PM850; IOMED). In the sham session, anodal tDCS was applied for $30 \mathrm{~s}$. At the offset of tDCS, the current was decreased in a ramp-like manner, a method shown to achieve a good level of blinding between sessions (Gandiga et al., 2006). Thus, we applied a current at a density of $0.08 \mathrm{~mA} / \mathrm{cm}^{2}$ and delivered a total current of $2 \mathrm{~mA} / \mathrm{cm}^{2}$. This is similar to Ferrucci et al. (2008) $(1.9 \mathrm{~mA} /$ $\mathrm{cm}^{2}$ ), is considered to be safe (Iyer et al., 2005), and is far below the threshold for tissue damage (Boggio et al., 2006).

TMS of M1. TMS was delivered using a 70-mm-diameter figure-ofeight coil (BiStim ${ }^{2}$ stimulator; Magstim). The coil was placed tangentially to the scalp with the handle pointed backward at a $45^{\circ}$ angle with respect to the anteroposterior axis (Fig. 1a). The motor "hot spot" for each of the targeted muscles was identified by single pulses of TMS de- livered at a slightly suprathreshold stimulus intensity. A frameless neuronavigation system (BrainSight; Rogue Research) was used to mark this spot after coregistration with a standard magnetic resonance image. Muscle relaxation was monitored by visual and audio feedback of the EMG signals.

TMS of the cerebellum and brainstem. TMS of the cerebellum was performed with a double-cone coil (110 $\mathrm{mm}$ mean diameter). The coil was centered over the right cerebellar cortex $3 \mathrm{~cm}$ lateral to the inion on the line joining the inion and the external auditory meatus (Fig. 1a). The current in the coil was directed downward, which induced upward current in the cerebellar cortex. For the brainstem measurement assessing pyramidal tract activation, the same coil was placed over the inion. These coil positions and current direction were found to be optimal in previous studies (Ugawa et al., 1995; Pinto and Chen, 2001; Daskalakis et al., 2004) and were marked for consistency using a felt pen on the scalp.

M1 excitability. First, we determined the resting motor threshold for the FDI muscle, which was defined as the minimum TMS intensity that evoked a motor-evoked potential (MEP) of $50 \mu \mathrm{V}$ in at least 5 of 10 trials in the resting target muscle (Rossini et al., 1994). Then, we established the stimulator intensity required to produce $1 \mathrm{mV}$ MEP responses. This intensity was retested after the intervention to assess changes in MEP amplitudes. Short intracortical inhibition (SICI) and short intracortical facilitation (ICF) were assessed using paired-pulse TMS with a subthreshold conditioning stimulus (CS) set at $80 \%$ of resting motor threshold, preceding a suprathreshold test stimulus (TS) to elicit $\sim 1 \mathrm{mV}$ MEPs. SICI was tested with a $2 \mathrm{~ms}$ interstimulus interval (ISI) and ICF with 10 ms (Table 1).

Cerebellar excitability. To assess cerebellar excitability before and after the interventions, we tested CBI. This was done by delivering a TMS CS over the right cerebellar cortex $5 \mathrm{~ms}$ before a TS over the left M1 (Ugawa et al., 1995; Werhahn et al., 1996; Pinto and Chen, 2001; Daskalakis et al., 2004) (Table 1, Fig. 1a). As described in previous studies, the intensity for cerebellar stimulation was set $5 \%$ below the brainstem active motor threshold (see below) (Ugawa et al., 1995; Pinto and Chen, 2001; Daskalakis et al., 2004). However, if the threshold was not observed at $80 \%$ of the stimulator output, $70 \%$ was used for cerebellar stimulation.

Brainstem excitability. To determine changes in brainstem excitability secondary to cerebellar tDCS, we assessed the brainstem motor threshold and MEP amplitudes of a trunk muscle ipsilateral to M1 stimulation, a measurement that has been suggested to reflect reticulospinal (RS) excitability (Ziemann et al., 1999).

The brainstem threshold for pyramidal tract activation was tested with a double-cone coil centered over the inion (Ugawa et al., 1995; Daskalakis et al., 2004). The threshold was defined as the nearest $5 \%$ stimulator output that elicited an MEP of $50 \mu \mathrm{V}$ in the preactivated FDI muscle in 5 of 10 trials (Table 1) (Ugawa et al., 1995; Pinto and Chen, 2001; Daskalakis et al., 2004). Of note, this form of stimulation is thought to activate descendent corticospinal axons reflecting spinal cord excitability rather than intrinsic brainstem excitability changes (Ugawa et al., 1994; Pinto and Chen, 2001). 
Table 1. Experiment 1

\begin{tabular}{|c|c|c|c|c|c|}
\hline Measure & Pretest & Preconditioning & Posttest & Postconditioning & Analysis \\
\hline MEP threshold & $\begin{array}{l}\text { 5/10 responses elicit MEPs of } \\
50 \mu \mathrm{V}\end{array}$ & & $\begin{array}{l}\text { 5/10 responses elicit MEPs of } \\
50 \mu \mathrm{V}\end{array}$ & & $\begin{array}{l}\text { Change in percentage of } \\
\text { stimulation output }\end{array}$ \\
\hline MEP response & $1 \mathrm{mV}$ response $(10)$ & & $\begin{array}{l}\text { Stimulation output used } \\
\text { during pre (10) }\end{array}$ & & $\begin{array}{l}\text { Comparison of pre and post } \\
\text { MEP amplitudes }\end{array}$ \\
\hline $\mathrm{SICl}$ & $1 \mathrm{mV}$ response (15) & $\begin{array}{l}80 \% \text { of MEP threshold, } 2 \text { ms } \\
\text { preceding test (15) }\end{array}$ & Adjusted $1 \mathrm{mV}$ response (15) & $\begin{array}{l}80 \% \text { of MEP threshold, } 2 \text { ms } \\
\text { preceding test (15) }\end{array}$ & Conditioning/test ratio \\
\hline ICF & $1 \mathrm{mV}$ response $(15)$ & $\begin{array}{l}80 \% \text { of MEP threshold, } 10 \mathrm{~ms} \\
\text { preceding test (15) }\end{array}$ & Adjusted $1 \mathrm{mV}$ response (15) & $\begin{array}{l}80 \% \text { of MEP threshold, } 10 \mathrm{~ms} \\
\text { preceding test (15) }\end{array}$ & Conditioning/test ratio \\
\hline CBI & $1 \mathrm{mV}$ response $(10)$ & $\begin{array}{l}5 \% \text { below brainstem threshold, } \\
5 \text { ms preceding test over the } \\
\text { ipsilateral cerebellum (10) }\end{array}$ & Adjusted $1 \mathrm{mV}$ response (10) & $\begin{array}{l}5 \% \text { below brainstem threshold, } \\
5 \text { ms preceding test over the } \\
\text { ipsilateral cerebellum (10) }\end{array}$ & Conditioning/test ratio \\
\hline Right M1 MEP response & $\begin{array}{l}1 \mathrm{mV} \text { response with contralateral } \\
\text { pectoral over right M1; } \\
\text { preactivation (15) }\end{array}$ & & $\begin{array}{l}\text { Adjusted } 1 \mathrm{mV} \text { response over } \\
\text { right M1; preactivation (15) }\end{array}$ & & $\begin{array}{l}\text { Comparison of pre and post MEP } \\
\text { amplitudes for pectoral } \\
\text { ipsilateral MEP response }\end{array}$ \\
\hline
\end{tabular}

TMS measures of motor cortex and cerebellar excitability. For each measure, an explanation is given for the test and conditioning pulses and the analysis used. Unless stated otherwise, the coil was placed on the hot spot of the left motor cortex (M1). The numbers within parentheses indicate the number of TMS pulses.

The RS activation was measured by applying single-pulse TMS over the right M1 while measuring EMG activity in the left and right preactivated pectoralis major muscles. With the aid of visual and audio feedback, subjects maintained an $\sim 400 \mu \mathrm{V}$ preactivation of both muscles. Using this technique, it is possible to observe MEPs in the contralateral muscle to the stimulation (corticospinal pathway) and MEPs in the ipsilateral pectoralis muscle 5-10 ms later than the contralateral response. It has been suggested that the ipsilateral MEP may represent the activation of the RS pathway, where the ipsilateral MEP latency delay corresponds to the synapse between the corticobulbar to RS neurons (Ziemann et al., 1999). Thus, we applied TMS at an intensity to elicit $\sim 1 \mathrm{mV}$ MEP amplitudes of the contralateral pectoralis muscle (left side, corticospinal pathway) and measured the responses on bilateral pectoralis muscles (Table 1).

In all paired-pulse TMS determinations, the intensities of the TS were adjusted to produce a comparable MEP amplitude size of $\sim 1 \mathrm{mV}$.

\section{Experiment 2: CBI recruitment curve}

Previous studies have evaluated CBI delivering only condition pulses over the cerebellum at 5\% below brainstem threshold (Ugawa et al., 1995; Pinto and Chen, 2001; Daskalakis et al., 2004). Since it is possible that this intensity elicits maximum recruitment of the cerebellar brain connections, increasing cerebellar excitability by tDCS would not result in additional M1 inhibition because of a ceiling effect. Therefore, to evaluate whether anodal tDCS enhances CBI, we performed a recruitment curve $\left(\mathrm{RC}_{\mathrm{CBI}}\right)$ of the conditioning pulse by decreasing the intensity of the cerebellar conditioning stimulation by $5 \%$ steps below brainstem threshold. If cerebellar excitability is increased as a result of the intervention, then lower condition stimuli intensities should result in a similar amount of CBI. Eight subjects participated in this second experiment (two women; mean age, $26 \pm 6$ years; range, $23-40$ years) in which CBI was measured as described previously (see above, Cerebellar excitability) using five different conditioning stimulation intensities $(-5,-10,-15,-20$, and $-25 \%$ below brainstem threshold) before and after $25 \mathrm{~min}$ of cerebellar anodal tDCS.

\section{Experiment 3: longevity of cathodal tDCS effect}

To assess the longevity of the cathodal tDCS effect, we measured CBI at 0 $\min$ (post 1), $30 \mathrm{~min}$ (post 2), and $50 \mathrm{~min}$ (post 3 ) after the cessation of tDCS stimulation. Six subjects (five women; mean age, $23 \pm 5$ years; range, $20-34$ years) were exposed to either $25 \mathrm{~min}$ of 1 or $2 \mathrm{~mA}$ cathodal stimulation in a double-blind, counterbalanced, crossover design. To determine that the effects observed in CBI were specific to the cerebellothalamo-cortical pathway described by previous investigations (Ugawa et al., 1991, 1995; Pinto and Chen, 2001; Daskalakis et al., 2004), we also evaluated whether CBI could be obtained at a shorter ISI of $3 \mathrm{~ms}$. Any changes in CBI using this ISI would suggest that tDCS influences deeper structures.

In addition, to expand on the measures of cortical excitability that may be influenced by cerebellar tDCS, we assessed changes in the M1 recruitment curve $\left(\mathrm{RC}_{\mathrm{M} 1}\right)$ of the FDI MEP before and after cathodal tDCS. The lowest intensity was set at the subject's motor threshold, and five different intensities were measured. The intensities were in $10 \%$ increments, and five TMS pulses were given for each intensity.

Finally, to extend the assessment of changes in brainstem excitability from experiment 1 , we measured ( 1 ) the amplitude of MEPs elicited from brainstem TMS delivered at 5\% above the subject's brainstem active threshold and (2) the recruitment curve of the subject's blink reflex $\left(\mathrm{RC}_{\mathrm{BR}}\right)$ before and after cathodal tDCS. The latter test involves stimulating the supraorbital nerve above the eye ipsilateral to the cerebellar stimulation side and assessing both orbicularis oculi muscles responses. Normally, an early ipsilateral response (R1) is followed by a later bilateral response (R2), which are both relayed through the V and VII cranial nerves and integrated by intrinsic brainstem circuits (Kimura, 1989). For this recruitment curve measurement, we initially found the threshold for the R1 response and then performed five pulses at each of five stimulus intensities at increments of $10 \%$ of the threshold.

\section{Data analysis (all experiments)}

The peak-to-peak MEP amplitude for each trial was measured. SICI, ICF, and CBI were calculated as the ratio of the conditioned to the test MEP amplitude for each subject (Table 1). Ratios $<1$ indicate inhibition, and ratios $>1$ indicate facilitation. MEP amplitudes during M1 TMS and RS measures were averaged in each subject for pre and post determinations and compared across sessions.

To determine the temporal difference between the ipsilateral and contralateral MEP when evaluating the RS measurement within experiment 1, the latency of each MEP peak relative to the TMS pulse was recorded and averaged for each subject in each session. To compare the EMG preactivation level during RS assessments, we first rectified and then averaged the EMG signals of the $150 \mathrm{~ms}$ before the TMS pulse. This was then compared using repeated-measures ANOVA $\left(\right.$ ANOVA $\left._{R M}\right)$ with factors muscle (contralateral, ipsilateral), session (anodal, cathodal, or sham), and time (pre, post).

The blink reflex within experiment 3 was assessed with four measures. First, the ipsilateral R1 response was measured by the amplitude and peak time of the brainstem-evoked response in the right orbicularis oculi muscle. The $\mathrm{R} 2$ response was measured by separately rectifying and summing the EMG data between 50 and $90 \mathrm{~ms}$ after stimulation, for both orbicularis oculi muscles (Kimura, 1989).

For experiment 1, separate ANOVA $\mathrm{RM}_{\text {mere }}$ used for MEP threshold, MEP amplitude, SICI, ICF, CBI, RS (contralateral and ipsilateral), and brainstem MEP threshold with factors session (anodal, cathodal, sham) and time (pre, post).

To evaluate the $\mathrm{RC}_{\mathrm{CBI}}$ (experiment 2), we used $\mathrm{ANOVA}_{\mathrm{RM}}$ to compare the effect of factors time (pre, post) and CS intensity $(-5,-10$, $-15,-20$, and $-25 \%$ below motor threshold). In addition, we used 
Table 2. Experiment 1: psychological measures

\begin{tabular}{llll}
\hline & Attention & Fatigue & Pain caused by tDCS \\
\hline Anodal & $5.5 \pm 0.5$ & $3.4 \pm 0.6$ & $2.5 \pm 0.4$ \\
Cathodal & $5.1 \pm 0.5$ & $2.4 \pm 0.3$ & $2.8 \pm 0.6$ \\
Sham & $5.6 \pm 0.4$ & $3.0 \pm 0.5$ & $2.3 \pm 0.5$ \\
ANOVA & $F=0.2, p=0.79$ & $F=1, p=0.39$ & $F=0.2, p=0.8$ \\
\hline
\end{tabular}

Values (mean \pm SEM) depict the subject's choice in a visual analog scale in which 1 represents poorest attention maximal fatigue, and pain and 7 represents maximal attention, least fatigue, and pain. F and $p$ values originate from separate ANOVAs for each measure comparing the anodal, cathodal, and sham sessions.

\section{Left M1 excitability}

ANOVA $_{\mathrm{RM}}$ revealed no significant changes in MEP threshold across session $\left(F_{(2,14)}=1.5 ; p=0.25\right)$, time $\left(F_{(2,14)}=2.9 ; p=\right.$ 0.13 ), or time $\times$ session interaction $\left(F_{(2,14)}=1.3 ; p=0.3\right.$ ) (Fig. $3 a)$. Similarly, mean MEP amplitudes elicited from $M 1$ did not differ over session $\left(F_{(2,14)}=0.09 ; p=0.9\right)$, time $\left(F_{(2,14)}=0.1 ; p=0.35\right)$, or time $\times$ session interaction $\left(F_{(2,14)}=0.08 ; p=0.9\right)$ (Fig. $\left.3 b\right)$.

Cerebellar tDCS did not result in changes in SICI or ICF across session, time, or session $\times$ time interaction $\left(\mathrm{ANOVA}_{\mathrm{RM}}\right.$ for SICI: session, $F_{(2,14)}=1.6, p=0.25$; time, $F_{(2,14)}=0.42, p=0.54$; interaction, $F_{(2,14)}=0.42, p=0.67$; ANO$\mathrm{VA}_{\mathrm{RM}}$ for ICF: session, $F_{(2,14)}=0.27, p=$ 0.77 ; time, $F_{(2,14)}=0.34, p=0.58$; interaction, $\left.F_{2,14)}=0.26, p=0.77\right)$ (Fig. $\left.3 c, d\right)$.
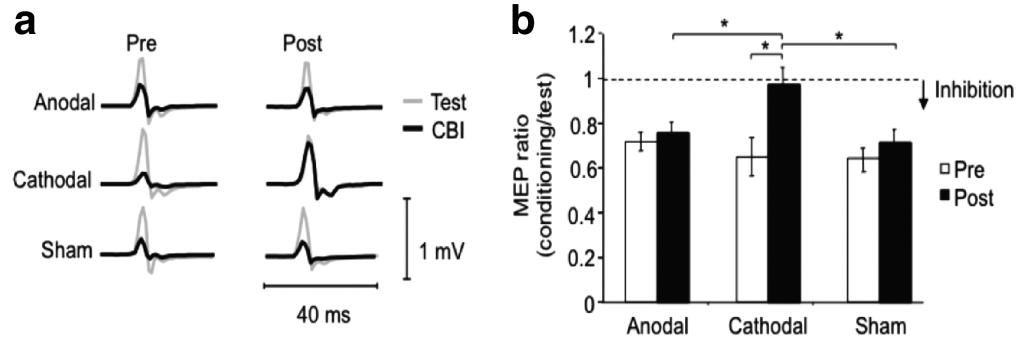

Figure 2. Single-subject and group CBI data. $\boldsymbol{a}$, Single MEP traces from tests (M1 stimulation; gray lines) and conditioned stimulation (cerebellar plus M1 stimulation; black lines) from a sample subject before (pre) and after (post) cerebellar stimulation is shown. Note that CBIMEPs showed the same amplitudes as the TS after cathodal tDCS, an effect not present after sham or anodal stimulation. $\boldsymbol{b}$, The amount of inhibition observed during pre (open bar) and post (filled bar) remained similar in the anodal and sham sessions. However, there was a significant decrease in inhibition from pre to post after cathodal stimulation. When comparing with anodal and sham, the amount of inhibition in post was significantly less in the cathodal session. ${ }^{*} p<0.02$. Data are means \pm SEM.

ANOVA $_{\mathrm{RM}}$ to compare the MEP amplitudes separately for pre and post $\mathrm{tDCS}$ stimulation with the factors responses (test, conditioned) and CS intensities $(-5,-10,-15,-20$, and $-25 \%$ below motor threshold).

The longevity of the cathodal tDCS effect (experiment 3 ) was assessed with $\mathrm{ANOVA}_{\mathrm{RM}}$, which compared CBI with factors time (pre, post 1 , post 2, post 3 ) and stimulation intensity $(1 \mathrm{~mA}, 2 \mathrm{~mA})$. The $\mathrm{RC}_{\mathrm{M} 1}$ and $\mathrm{RC}_{\mathrm{BR}}$ were evaluated by separate $\mathrm{ANOVA}_{\mathrm{RM}}$, which compared the effect of time (pre, post 1), stimulation intensity ( $1 \mathrm{~mA}, 2 \mathrm{~mA})$, and test intensity $(100 \%, 110 \%, 120 \%, 130 \%$, and $140 \%$ of threshold). The MEP amplitude resulting from TMS at the inion was assessed with an ANOVA $_{R M}$ comparing time (pre, post 1 ) and stimulation intensity ( $1 \mathrm{~mA}, 2 \mathrm{~mA}$ ).

When significant differences were found, post hoc analysis was performed using paired $t$ tests. Data are expressed as mean \pm SEM, and effects were considered significant if $p \leq 0.05$.

\section{Results}

Experiment 1: modulation of cerebellar excitability by tDCS All subjects completed the three sessions without complications. The subject's self-reported ratings of attention, fatigue, and perceived pain were not significantly different across the three sessions $\left(F_{(2,14)}\right.$ $<1 ; p>0.39$ ) (Table 2). All measures were completed within $25 \mathrm{~min}$ of the cessation of $\mathrm{tDCS}$.

\section{Cerebellar excitability}

tDCS applied over the cerebellum elicited modulation of CBI (Fig. 2a). ANOVA $_{\mathrm{RM}}$ revealed a significant effect of tDCS on CBI (conditioning MEP/test MEP) over time (pre, post; $F_{(2,14)}=17.4$; $p=0.005)$ and time $\times$ session interaction $\left(F_{(2,14)}=11.8 ; p=\right.$ $0.005)$ and a trend toward significance across sessions (anodal, cathodal, sham; $F_{(2,14)}=3.2 ; p=0.07$ ) (Fig. $2 b$ ). Paired $t$ tests revealed that during the cathodal session there was a significant decrease in CBI from pre $(0.65 \pm 0.07)$ to post $\left(0.97 \pm 0.07 ; t_{(7)}=\right.$ $5.8 ; p<0.001$ ) (Fig. 2b). In addition, there was significantly less CBI within the cathodal session's post phase compared with the anodal $(0.75 \pm 0.05)$ and $\operatorname{sham}\left(0.71 \pm 0.06 ; t_{(7)}>2.5 ; p<0.02\right)$ sessions (Fig. 2b). pectoralis muscles. As a reminder, the pectoralis muscle ipsilateral to M1 stimulation was also ipsilateral to the targeted cerebellar tDCS hemisphere. The mean peak latency difference between ipsilateral and contralateral MEPs was $7.7 \pm 1$ $\mathrm{ms}$, in which the ipsilateral responses were always later than the contralateral MEPs (contralateral MEP, $13.1 \pm 0.45 \mathrm{~ms}$; ipsilateral MEP, $20.8 \pm 1.3 \mathrm{~ms}$; paired $t$ test: $\left.t_{(7)}=7.4, p=0.0005\right)$. During RS measurements, there was no difference in EMG preactivation $\left(\mathrm{ANOVA}_{\mathrm{RM}}\right.$ : muscle, $F_{(1,7)}=5.7, p=0.055$; session, $F_{(2,12)}=$ $0.6, p=0.57$; time, $F_{(1,6)}=0.32, p=0.6$; interactions, $F_{(2,12)}<1.8$, $p>0.2)$. MEP amplitudes were not influenced by the tDCS across session, time, or session $\times$ time interaction in either the contralateral $\left(\right.$ ANOVA $_{\mathrm{RM}}$ : session, $F_{(2,14)}=0.09, p=0.9$; time, $F_{(2,14)}=2.8, p=$ 0.14 ; interaction, $\left.F_{(2,14)}=0.79, p=0.47\right)$ or ipsilateral $\left(\mathrm{ANOVA}_{\mathrm{RM}}\right.$ : session, $F_{(2,14)}=1, p=0.38$; time, $F_{(2,14)}=1.4, p=0.27$; interaction, $F_{(2,14)}=0.45, p=0.65$ ) (Fig. $\left.4 b\right)$ pectoralis muscle.

\section{Experiment 2: CBI recruitment curve}

Anodal tDCS applied over the cerebellum lead to changes in the $\mathrm{RC}_{\mathrm{CBI}}$ (Fig. 5). The mean brainstem motor threshold from which conditioning stimulation intensities were set was $70 \pm 13 \%$ of the stimulator output. The test stimulation MEP amplitudes were not significantly different between pre and post tDCS stimulation (1.1 \pm 0.2 and $1.1 \pm 0.2 \mathrm{mV}$, respectively; paired $t$ test: $t_{(7)}=0.5$, $p=0.3)$. ANOVA $_{\mathrm{RM}}$ comparing the MEP amplitudes during test versus conditioned responses across CS intensity before tDCS revealed no significant effect for responses $\left(F_{(1,7)}=3 ; p=0.12\right)$ or CS intensity $\left(F_{(4,28)}=2 ; p=0.12\right)$; however, the interaction between responses and CS intensity was significant $\left(F_{(4,28)}=7.7\right.$; $p=0.005$ ). Paired $t$ tests revealed significant differences between test and conditioned responses only at CS intensities $-5 \%$ (test, $1.1 \pm 0.2$; conditioned, $0.68 \pm 0.1 ; t_{(7)} 3.3 ; p=0.01$, two-tailed) and $-10 \%$ (test, $1 \pm 0.17$; conditioned, $0.74 \pm 0.09 ; t_{(7)}=3 ; p=$ 0.02 ), suggesting a lack of CBI when the CS intensities were $-15 \%$ of the brainstem threshold or less. To the contrary, a similar ANOVA $_{\mathrm{RM}}$ performed for the MEP amplitudes after tDCS 
a M1 MEP threshold

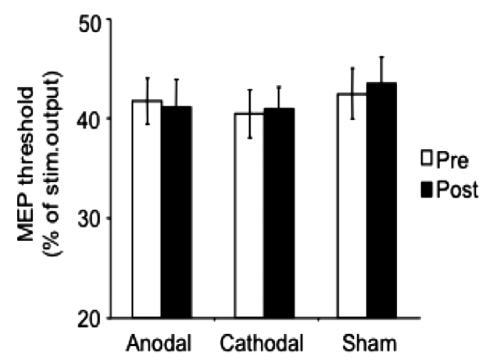

C $\mathrm{SICl}$

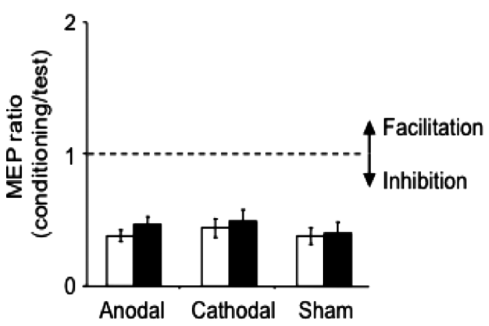

b M1 MEP amplitude

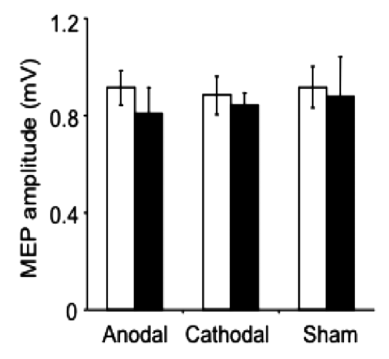

d ICF

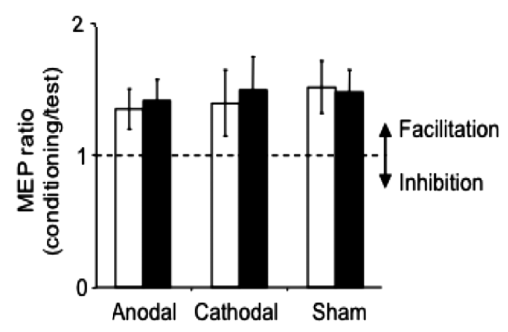

Figure 3. Measures of left M1 excitability. $\boldsymbol{a}$, MEP threshold; $\boldsymbol{b}$, MEP amplitude; $\boldsymbol{c}, \mathrm{SICl} ; \boldsymbol{d}$, ICF. For all measures, there were no significant differences for session (anodal, cathodal, sham tDCS) or time (pre, post). Open bar, Pre; filled bar, post. Data are means \pm SEM.
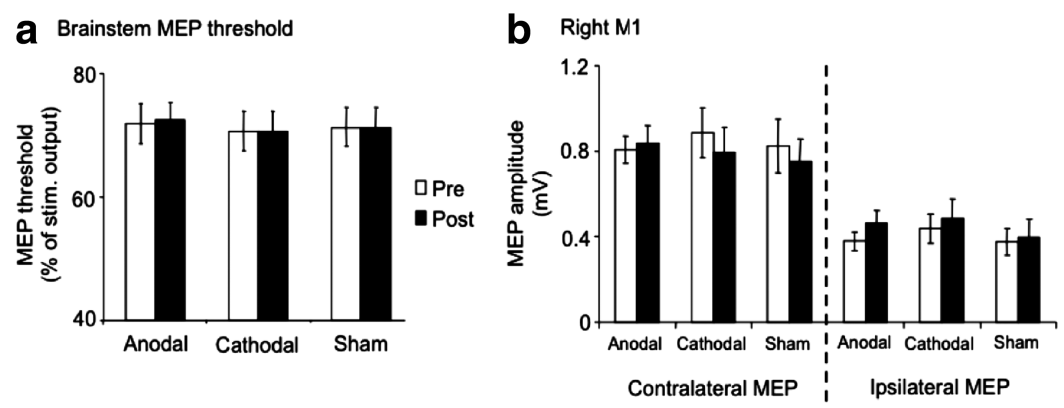

Figure 4. Measures of brainstem excitability. $\boldsymbol{a}$, There were no significant differences for session (anodal, cathodal, sham) or time (pre, post) for brainstem MEP thresholds. $\boldsymbol{b}$, There were no significant differences for session (anodal, cathodal, sham) or time (pre, post) for the contralateral (corticospinal) or ipsilateral (RS) MEP amplitudes originating from the right M1. Open bar, Pre; filled bar, post. Data are means \pm SEM.

revealed a significant difference for test versus conditioned responses $\left(F_{(1,7)}=6.3 ; p=0.04\right)$, but there was no significant effect for CS intensity $\left(F_{(4,28)}=0.8 ; p=0.75\right)$ or the interaction between responses and CS intensity $\left(F_{(4,28)}=0.4 ; p=0.8\right)$, indicating that CBI after anodal tDCS was present in all condition stimulation intensities tested. In addition, when comparing $\mathrm{RC}_{\mathrm{CBI}}$ before and after tDCS, we found significant differences across CS intensity and in the interaction time $\times$ CS intensity $\left(\mathrm{ANOVA}_{\mathrm{RM}}: F_{(4,28)}=5.7, p=0.002\right.$, and $F_{(4,28)}=3.7, p=0.015$, respectively). ANOVA $\mathrm{RM}_{\mathrm{RM}}$ also showed a trend toward significance for time (pre vs post: $F_{(1,7)}=4.2 ; p=0.08$ ) (Fig. 5). Post hoc paired $t$ tests revealed that at the lowest CS intensity, CS $-25 \%$ below brainstem threshold, there was a significant difference between CBI before and after anodal tDCS $(1.14 \pm 0.12$ and $0.82 \pm$ 0.03 , respectively; $\left.t_{(7)}=3.2 ; p=0.008\right)$. There was also a trend toward significance at the second lowest CS intensity (CS $-20 \%$ below brainstem threshold; $t_{(7)}=1.6 ; p=0.08$ ) (Fig. 5).

\section{Experiment 3: longevity of cathodal tDCS effect}

All subjects completed the two sessions without complications. The subject's self-reported ratings of attention, fatigue, and per-

ceived pain were not significantly different across the two sessions $\left(t\right.$ test: $t_{(5)}<1$; $p>0.3$ ) (Table 3).

Similar to experiment $1,2 \mathrm{~mA}$ cathodal tDCS led to a decrease in the magnitude of CBI, which was sustained for at least $30 \mathrm{~min}$ in the absence of changes after $1 \mathrm{~mA}$ tDCS. ANOVA RM showed a significant main effect of time $\left(F_{(3,15)}=6.6 ; p=0.05\right)$, stimulation intensity $\left(F_{(1,5)}=12 ; p=0.02\right)$, and interaction between time $X$ stimulation intensity $\left(F_{(3,15)}=3.5 ; p=0.04\right)$ (Fig. 6a). Post hoc paired $t$ tests revealed a significant difference between 1 and $2 \mathrm{~mA}$ intensity sessions at post $1(0.62 \pm 0.07$ and $1.0 \pm 0.1$, respectively; $t_{(5)}=4.3 ; p=0.01$, twotailed) and post $2(0.66 \pm 0.07$ and $0.96 \pm$ 0.08 , respectively; $t_{(5)}=4 ; p=0.01$ ) (Fig. $6 a$ ). In addition, in the $2 \mathrm{~mA}$ session, there was a significant decrease in CBI between pre and post $1\left(t_{(5)}=5 ; p=0.004\right)$ and post $2\left(t_{(5)}=3.7 ; p=0.01\right)$, but not for post $3\left(t_{(5)}=1.2 ; p=0.3\right)$.

ANOVA $_{\mathrm{RM}}$ revealed a significant effect on $\mathrm{RC}_{\mathrm{M} 1}$ for test intensity $\left(F_{(4,20)}=38\right.$; $p=0.0005$ ) (Fig. 6b,c), but not for time $\left(F_{(1,5)}=0.7 ; p=0.4\right)$, stimulation intensity $\left(F_{(1,5)}=0.5 ; p=0.5\right)$, or the interactions $\left(F_{(4,20)}<0.4 ; p>0.6\right)$.

ANOVA $_{\mathrm{RM}}$ assessing MEP amplitudes from TMS over the inion showed no significant differences for time $\left(F_{(1,5)}=0.3\right.$; $p=0.6)$, stimulation intensity $\left(F_{(1,5)}=\right.$ $0.1 ; p=0.1)$, or the interaction between time $\times$ stimulation intensity $\left(F_{(1,5)}=0.4\right.$; $p=0.1$ ).

When assessing $\mathrm{RC}_{\mathrm{BR}}$, only the $\mathrm{R} 1$ amplitude revealed a main effect of stimulation intensity in an $\mathrm{ANOVA}_{\mathrm{RM}}\left(F_{(4,20)}=5.4\right.$; $p=0.004)$ (Fig. $6 d$ ). In contrast, R1 peak time and ipsilateral and contralateral R2 amplitude and peak time showed no significant difference for stimulation intensity $\left(F_{(4,20)}<2.8 ; p>0.05\right)$, time $\left(F_{(1,5)}<1.9\right.$; $p>0.15)$, test intensity $\left(F_{(1,5)}<1 ; p>0.35\right)$, and their interactions $\left(F_{(4,20)}<2.3 ; p>0.09\right)$ (Table 4$)$.

Finally, we could not obtain CBI either before or after tDCS when the ISI between the conditioning and test pulse was $3 \mathrm{~ms}$ (Table 3).

\section{Discussion}

The main finding of this study was the ability of tDCS to modulate cerebellar excitability in humans. In particular, we found that cathodal tDCS can decrease and anodal tDCS can increase the inhibitory tone the cerebellum exerts over the primary motor cortex. In addition, we found that cathodal tDCS effects last up to $30 \mathrm{~min}$ after the cessation of stimulation and are present with a stimulation intensity of $2 \mathrm{~mA}$.

Previous studies have investigated the effects of noninvasive cerebellar stimulation (Oliveri et al., 2005; Fierro et al., 2007; Koch et al., 2008; Langguth et al., 2008). These investigations have used inhibitory repetitive TMS, to induce a "virtual lesion" of the cerebellum and evaluate the physiological effects on excit- 


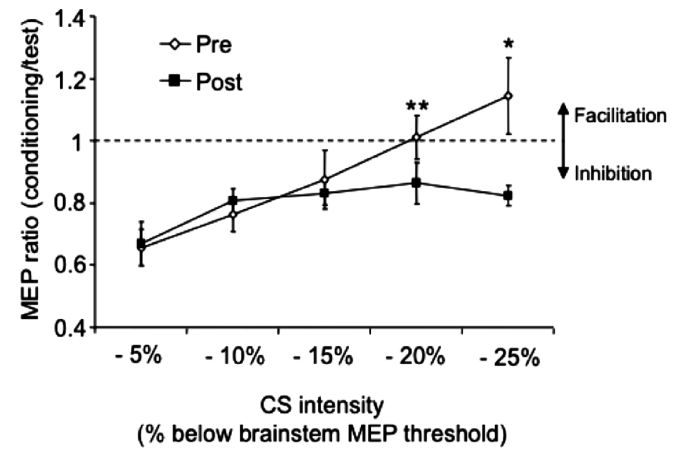

Figure 5. Experiment 2: (BI recruitment curve. To assess the effect of cerebellar anodal stimulation, we performed a $\mathrm{RC}_{\mathrm{CB}}$ of the conditioning TMS pulse intensities (cerebellar TMS) before (pre; open diamonds) and after (post; filled squares) tDCS. During pre, as the CS intensity is reduced in $5 \%$ step decrements of the stimulator output (from -5 to $-25 \%$ ), the amount of CBI decreases. After 25 min of anodal tDCS, CBI remains present even at condition stimulus intensities that previously did not elicit CBI $(-20$ and $-25 \%$ below brainstem threshold). ${ }^{*} p=0.008 ;{ }^{* *} p=0.08$. Data are means \pm SEM.

Table 3. Experiment 3

\begin{tabular}{|c|c|c|}
\hline & $1 \mathrm{~mA}$ & $2 \mathrm{~mA}$ \\
\hline \multicolumn{3}{|c|}{ Psychological measures ${ }^{a}$} \\
\hline Attention & $6.5 \pm 0.3$ & $6.7 \pm 0.3$ \\
\hline Fatigue & $1.7 \pm 0.2$ & $1.8 \pm 0.2$ \\
\hline Pain & $3.8 \pm 0.2$ & $3.3 \pm 0.6$ \\
\hline \multicolumn{3}{|c|}{$\mathrm{CB}^{b}$ (conditioning/test) $-3 \mathrm{~ms} \mid \mathrm{SI}$} \\
\hline Pre & $1.07 \pm 0.8$ & $1.08 \pm 0.1$ \\
\hline Post 1 & $1.05 \pm 0.1$ & $1.1 \pm 0.2$ \\
\hline Post 2 & $1.03 \pm 0.04$ & $1.1 \pm 0.1$ \\
\hline Post 3 & $1.05 \pm 0.03$ & $1.2 \pm 0.2$ \\
\hline
\end{tabular}

${ }^{a}$ Values depict the subject's choice in a visual analog scale in which 1 represents poorest attention, maximal fatigue, and pain and 7 represents maximal attention, least fatigue, and pain across stimulation intensity $(1 \mathrm{~mA}, 2 \mathrm{~mA}$ cathodal tD(S).

${ }^{b}$ Values represent CBI with an $|S|$ of $3 \mathrm{~ms}$ over time (pre, post 1, post 2, post 3$)$ and stimulation intensity $(1 \mathrm{~mA}, 2 \mathrm{~mA})$.

\section{Table 4. Experiment 3}

\begin{tabular}{|c|c|c|c|c|}
\hline & $1 \mathrm{~mA}$ pre & $1 \mathrm{~mA}$ post 1 & $2 \mathrm{~mA}$ pre & $2 \mathrm{~mA}$ post 1 \\
\hline \multicolumn{5}{|c|}{ Blink reflex: R1 peak time ${ }^{a}$} \\
\hline $100 \%$ & $8.5 \pm 0.4$ & $8.8 \pm 0.2$ & $8.6 \pm 0.3$ & $8.7 \pm 0.2$ \\
\hline $110 \%$ & $8.1 \pm 0.5$ & $8.4 \pm 0.3$ & $8.4 \pm 0.2$ & $8.5 \pm 0.3$ \\
\hline $120 \%$ & $8.0 \pm 0.6$ & $8.2 \pm 0.5$ & $8.5 \pm 0.2$ & $8.3 \pm 0.2$ \\
\hline $130 \%$ & $8.2 \pm 0.5$ & $8.5 \pm 0.3$ & $8.3 \pm 0.4$ & $8.5 \pm 0.2$ \\
\hline $140 \%$ & $8.0 \pm 0.3$ & $8.0 \pm 0.5$ & $8.3 \pm 0.3$ & $8.3 \pm 0.3$ \\
\hline \multicolumn{5}{|c|}{ Blink reflex: R2 ipsilateral ${ }^{b}$} \\
\hline $100 \%$ & $7.5 \pm 2.5$ & $7.9 \pm 2.0$ & $6.3 \pm 0.6$ & $6.8 \pm 1.0$ \\
\hline $110 \%$ & $7.9 \pm 2.7$ & $7.3 \pm 1.6$ & $6.9 \pm 0.8$ & $6.8 \pm 1.0$ \\
\hline $120 \%$ & $8.3 \pm 2.8$ & $7.6 \pm 1.8$ & $7.6 \pm 0.9$ & $7.5 \pm 1.2$ \\
\hline $130 \%$ & $8.9 \pm 2.6$ & $7.6 \pm 1.6$ & $8.1 \pm 0.9$ & $7.1 \pm 1.0$ \\
\hline $140 \%$ & $8.1 \pm 2.8$ & $7.7 \pm 1.5$ & $8.7 \pm 1.1$ & $8.0 \pm 1.1$ \\
\hline \multicolumn{5}{|c|}{ Blink reflex: R2 contralateral' } \\
\hline $100 \%$ & $5.4 \pm 1.7$ & $5.7 \pm 1.7$ & $4.4 \pm 0.6$ & $4.6 \pm 1.1$ \\
\hline $110 \%$ & $5.4 \pm 1.7$ & $5.6 \pm 1.9$ & $4.8 \pm 0.9$ & $4.7 \pm 1.0$ \\
\hline $120 \%$ & $6.3 \pm 2.2$ & $5.9 \pm 1.9$ & $5.2 \pm 0.9$ & $5.2 \pm 1.1$ \\
\hline $130 \%$ & $6.7 \pm 2.3$ & $6.0 \pm 1.9$ & $5.6 \pm 0.8$ & $5.2 \pm 1.3$ \\
\hline $140 \%$ & $6.2 \pm 2.0$ & $5.9 \pm 1.9$ & $5.5 \pm 1.1$ & $5.4 \pm 1.2$ \\
\hline
\end{tabular}

${ }^{a}$ A recruitment curve assessed the blink reflex at five intensities $(100,110,120,130$, and $140 \%$ of brainstem evoked potential) over time (pre, post 1) and stimulation intensity (1 mA, $2 \mathrm{~mA})$. Values represent the time point (in milliseconds) of the peak evoked potential.

${ }^{b}$ Values indicate the rectified and summed EMG data between 50 and $90 \mathrm{~ms}$ after stimulation in the ipsilateral orbicularis oculi muscle (mean \pm SEM).

'Values represent a similar measure to R2 ipsilateral but with the contralateral orbicularis oculi muscle (mean \pm SEM). ability of the primary motor cortex. Unfortunately, the effects described in these studies are indirect and inconsistent, and none of them measured the cerebellar motor connections (Oliveri et al., 2005; Fierro et al., 2007; Koch et al., 2008; Langguth et al., 2008). In the present study, we aimed to both inhibit and enhance cerebellar excitability using tDCS and determine the effects by measuring changes in cerebello-motor connections.

The normal inhibitory tone the cerebellum exerts over the primary motor cortex, CBI, can be assessed using paired-pulse TMS (Ugawa et al., 1995; Pinto and Chen, 2001; Daskalakis et al., 2004). In these studies, a conditioning pulse delivered over one cerebellar cortex 5-7 ms before a test pulse over the contralateral M1 results in a decrease of the motor-evoked potential amplitude relative to single-pulse TMS over the same M1. The decreased MEP amplitude reflects inhibition of M1, an effect attributed to activation of Purkinje cells resulting in inhibition of the dentate nucleus, which in turn has a disynaptic excitatory connection through the ventral thalamus to the contralateral M1 (Ugawa et al., 1995; Pinto and Chen, 2001; Daskalakis et al., 2004; Reis et al., 2008). Therefore, it is possible to probe the excitability level of the cerebellum by testing directly the amount of CBI.

Our results suggest that tDCS modulates Purkinje cell excitability. Application of cathodal tDCS, known to decrease excitability (Purpura and McMurtry, 1965; Nitsche and Paulus, 2000), resulted in a reduction of CBI. This is likely attributable to reduced Purkinje cell excitability resulting in the conditioning TMS pulse not activating these cells and consequently not causing inhibition of the excitatory connection between the dentate nucleus and M1. On the contrary, increased Purkinje cell excitability after anodal tDCS, a form of stimulation that increases excitability (Purpura and McMurtry, 1965; Nitsche and Paulus, 2000), can explain the facilitation of CBI. This was determined by persistent M1 inhibition even when the cerebellum was conditioned with low TMS intensities. Thus, anodal tDCS would allow lowintensity TMS pulses to cause activation of the Purkinje cells and, subsequently, inhibition of the dentate nucleus and M1 excitatory connection. Importantly, these findings cannot be explained by simple passage of time, as sham stimulation did not elicit any significant changes, nor to nonspecific effects of the stimulation on attention, fatigue, or pain (Table 2). In addition, the subjects were not able to determine what kind of stimulation they received in each session.

The findings of the present study suggest that tDCS exerted its effects focally over the cerebellum without affecting brainstem or corticomotor excitability. First, we did not find any changes in brainstem motor thresholds or brainstem MEP amplitudes, as determined by TMS applied over the inion (Ugawa et al., 1994, 1995; Pinto and Chen, 2001; Daskalakis et al., 2004). However, these measures may reflect spinal cord excitability rather than brainstem, since it has been suggested that TMS over the inion activates descending corticospinal axons (Ugawa et al., 1994; Pinto and Chen, 2001). Nonetheless, we did not observe changes in the ipsilateral pectoralis MEP amplitudes, suggested to reflect RS excitability (Ziemann et al., 1999). In addition, we also failed to observe changes in the recruitment curve of either the short ipsilateral (R1) or long bilateral (R2) eye-blink reflex, which is integrated via intrinsic brainstem circuits (Kimura, 1989). Despite all these negative findings, it is important to note that subtle changes in brainstem excitability may still be occurring, but we were not able to detect them with the measures implemented.

Interestingly, we did not find any significant changes in M1 excitability, as determined by motor threshold, MEP amplitudes, MEP recruitment curves, intracortical excitability, probed with 

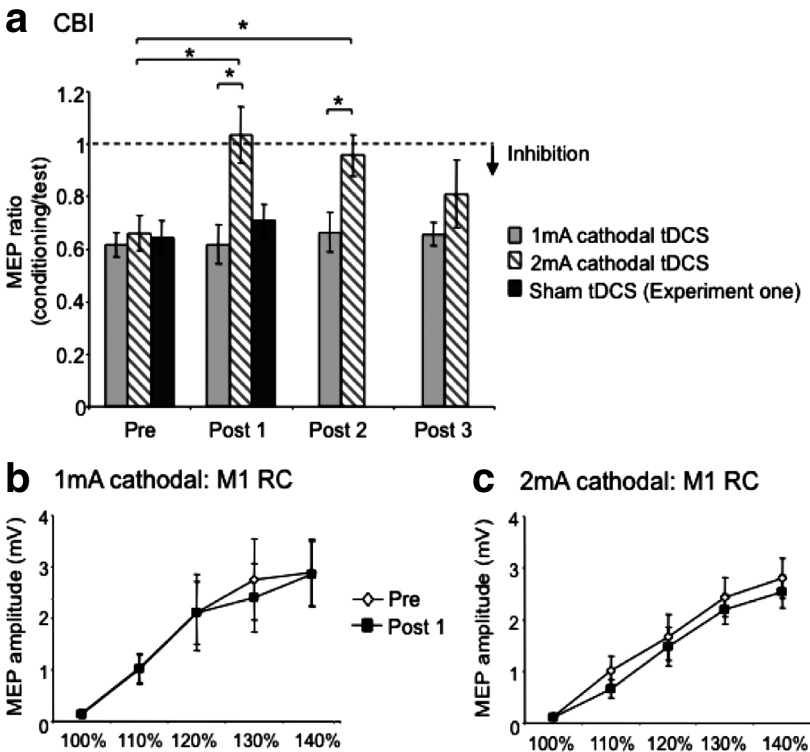

C $2 \mathrm{~mA}$ cathodal: $\mathrm{M} 1 \mathrm{RC}$
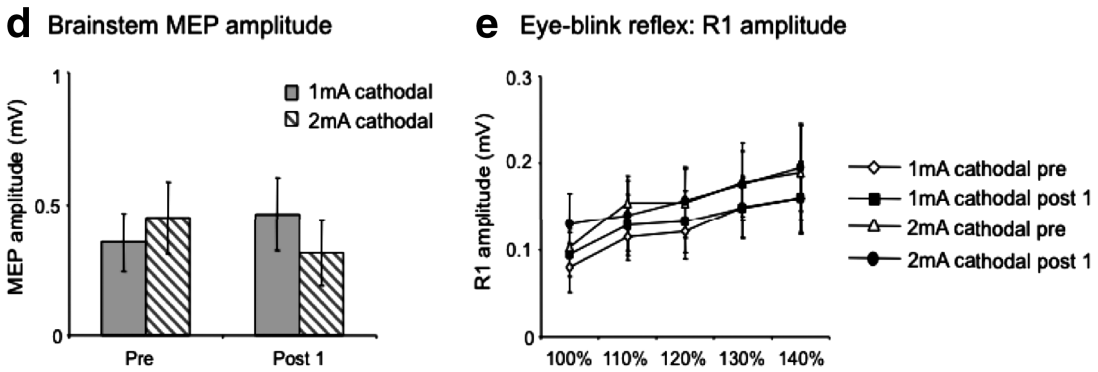

Figure 6. Experiment three: Longevity of cathodal tDCS effect. $\boldsymbol{a}, \mathrm{CBI}$. The effect of $1 \mathrm{~mA}$ (light gray bar) and $2 \mathrm{~mA}$ (gray striped bar) cathodal tDCS was measured across time (pre, post 1, post 2, post 3). Note the significant decrease in CBI after $2 \mathrm{~mA}$ cathodal tDCS relative to $1 \mathrm{~mA}$ stimulation intensity in post 1 and post 2 measures. In addition, during the $2 \mathrm{~mA}$ cathodal $\mathrm{tDCS}$ session, there was a significant decrease in CBI from pre to post 1 and post 2, but not for post 3 . These indicate the importance of stimulation intensity and the duration of the effects. Sham stimulation (results from experiment 1; dark gray bar) is shown to demonstrate the similar $\mathrm{CB}$ magnitude with $1 \mathrm{~mA}$ stimulation. ${ }^{*} p<0.01 . \boldsymbol{b}, \boldsymbol{c}, 1 \mathrm{~mA}: \mathrm{RC}_{\mathrm{M} 1} / 2 \mathrm{~mA}$ : $\mathrm{RC}_{\mathrm{M} 1}$. No significant changes in the $\mathrm{RC}_{\mathrm{M} 1}$ were observed after 1 or $2 \mathrm{~mA}$ stimulation. The $x$-axis indicates the TMS intensity relative to the MEP threshold. RC, Recruitment curve. $\boldsymbol{d}$, Brainstem MEP amplitude. No significant changes in the MEP amplitudes originating from inion TMS ( $5 \%$ above threshold) were observed after 1 or $2 \mathrm{~mA}$ tDCS. $e$, Eye-blink reflex: $\mathrm{R} 1$ amplitude. No significant changes in the $\mathrm{RC}_{\mathrm{BR}}$ were observed after 1 or $2 \mathrm{~mA}$ stimulation. The $x$-axis indicates the nerve stimulation intensity relative to the $R 1$ threshold. Data are means \pm SEM.

SICI and ICF, after cerebellar tDCS. Because these measures were all taken within $25 \mathrm{~min}$ after the cessation of tDCS, and the cathodal effect on CBI was still observable at $30 \mathrm{~min}$ (experiment 3 ), it is unlikely that the lack of effects on these TMS measures was attributable to the tDCS effects subsiding before their assessment. The lack of effects of cerebellar tDCS on M1 excitability contrasts to previous low-frequency rTMS studies in which cerebellar cortex stimulation resulted in either increase (Oliveri et al., 2005; Koch et al., 2008) or decrease intracortical excitability (Fierro et al., 2007; Langguth et al., 2008). A possible explanation to this discrepancy is that unlike TMS, tDCS did not cause direct depolarization of the stimulated neurons but rather a change in cell polarity (Purpura and McMurtry, 1965). Thus, tonic changes in Purkinje cell excitability at rest may not lead to direct activation of the cerebello-thalamo-cortical pathway. This would be in contrast to the rTMS studies in which repetitive depolarization of Purkinje cells may lead to the engagement of the cerebello-thalamocortical pathway and the consequent changes in contralateral M1 excitability (Oliveri et al., 2005; Fierro et al., 2007; Koch et al., 2008; Langguth et al., 2008). Alternatively, it is also possible that changes in M1 excitability were not detected because of SICI and ICF being assessed only with one ISI.
At present, the cellular mechanism by which tDCS influences cerebellar excitability is not known. The modulation of Purkinje cell activity is dependent on synaptic processes involving calcium and sodium channels, GABA, and AMPA receptor modulation (Shepherd, 2004). Although the underlying neurophysiology of tDCS is not completely understood, the modulation of sodium and calcium channels, NMDA receptors (Nitsche et al., 2003b), brain-derived neurotrophic factor (Cheeran et al., 2008), and cholinergic neuromodulation through acetylcholine (Kuo et al., 2007) have been described as contributing mechanisms. Additional research is required to understand how these mechanisms affect the cerebellum; however, it may be possible that tDCS alters the tonic excitability of Purkinje cells through modulation of calcium and sodium channels.

The present results suggest that tDCS may have potential to be used as a rehabilitation intervention to enhance motor function in patients with cerebellar lesions and CBI may have potential to be used as a neurophysiological measure to assess cerebellar excitability changes. Furthermore, these interventions could be used to advance our knowledge of the cerebellar function in healthy humans by affecting processes described in animal studies [e.g., the recent demonstration in cats that Purkinje cell activity reflects the operation of an internal model based on memory of its previous motion (Cerminara et al., 2009)].

In conclusion, tDCS applied over the cerebellum modulates cerebellar excitability in a polarity-specific manner, as evidenced by changes in the output of the cerebellum, CBI. This suggests that cerebellar tDCS could become an intervention with significant potential applications for motor control studies and to investigations in patients with neurological conditions. In addition, cerebellar tDCS may have potential to be used as a rehabilitation intervention to enhance motor function in patients with cerebellar lesions.

\section{References}

Battaglia F, Quartarone A, Ghilardi MF, Dattola R, Bagnato S, Rizzo V, Morgante L, Girlanda P (2006) Unilateral cerebellar stroke disrupts movement preparation and motor imagery. Clin Neurophysiol 117:1009-1016.

Boggio PS, Ferrucci R, Rigonatti SP, Covre P, Nitsche M, Pascual-Leone A, Fregni F (2006) Effects of transcranial direct current stimulation on working memory in patients with Parkinson's disease. J Neurol Sci 249:31-38.

Cerminara NL, Apps R, Marple-Horvat DE (2009) An internal model of a moving visual target in the lateral cerebellum. J Physiol 587:429-442.

Cheeran B, Talelli P, Mori F, Koch G, Suppa A, Edwards M, Houlden H, Bhatia K, Greenwood R, Rothwell JC (2008) A common polymorphism in the brain-derived neurotrophic factor gene (BDNF) modulates human cortical plasticity and the response to rTMS. J Physiol 586:5717-5725.

Chen H, Hua SE, Smith MA, Lenz FA, Shadmehr R (2006) Effects of human 
cerebellar thalamus disruption on adaptive control of reaching. Cereb Cortex 16:1462-1473.

Daskalakis ZJ, Paradiso GO, Christensen BK, Fitzgerald PB, Gunraj C, Chen R (2004) Exploring the connectivity between the cerebellum and motor cortex in humans. J Physiol 557:689-700.

Ferrucci R, Marceglia S, Vergari M, Cogiamanian F, Mrakic-Sposta S, Mameli F, Zago S, Barbieri S, Priori A (2008) Cerebellar transcranial direct current stimulation impairs the practice-dependent proficiency increase in working memory. J Cogn Neurosci 20:1687-1697.

Fierro B, Giglia G, Palermo A, Pecoraro C, Scalia S, Brighina F (2007) Modulatory effects of $1 \mathrm{~Hz}$ rTMS over the cerebellum on motor cortex excitability. Exp Brain Res 176:440-447.

Gandiga PC, Hummel FC, Cohen LG (2006) Transcranial DC stimulation (tDCS): a tool for double-blind sham-controlled clinical studies in brain stimulation. Clin Neurophysiol 117:845-850.

Gironell A, Kulisevsky J, Lorenzo J, Barbanoj M, Pascual-Sedano B, Otermin $P$ (2002) Transcranial magnetic stimulation of the cerebellum in essential tremor: a controlled study. Arch Neurol 59:413-417.

Hummel F, Celnik P, Giraux P, Floel A, Wu WH, Gerloff C, Cohen LG (2005) Effects of non-invasive cortical stimulation on skilled motor function in chronic stroke. Brain 128:490-499.

Imamizu H, Miyauchi S, Tamada T, Sasaki Y, Takino R, Putz B, Yoshioka T, Kawato M (2000) Human cerebellar activity reflecting an acquired internal model of a new tool. Nature 403:192-195.

Iyer MB, Mattu U, Grafman J, Lomarev M, Sato S, Wassermann EM (2005) Safety and cognitive effect of frontal DC brain polarization in healthy individuals. Neurology 64:872-875.

Kelly RM, Strick PL (2003) Cerebellar loops with motor cortex and prefrontal cortex of a nonhuman primate. J Neurosci 23:8432-8444.

Kimura J (1989) Electordiagnosis in diseases of nerve and muscle: principles and practice. Philadelphia: F. A. Davis.

Koch G, Mori F, Marconi B, Codeca C, Pecchioli C, Salerno S, Torriero S, Lo Gerfo E, Mir P, Oliveri M, Caltagirone C (2008) Changes in intracortical circuits of the human motor cortex following theta burst stimulation of the lateral cerebellum. Clin Neurophysiol 119:2559-2569.

Kuo MF, Grosch J, Fregni F, Paulus W, Nitsche MA (2007) Focusing effect of acetylcholine on neuroplasticity in the human motor cortex. J Neurosci 27:14442-14447.

Langguth B, Eichhammer P, Zowe M, Landgrebe M, Binder H, Sand P, Hajak G (2008) Modulating cerebello-thalamocortical pathways by neuronavigated cerebellar repetitive transcranial stimulation (rTMS). Neurophysiol Clin 38:289-295.

Liepert J, Kucinski T, Tuscher O, Pawlas F, Baumer T, Weiller C (2004) Motor cortex excitability after cerebellar infarction. Stroke 35: $2484-2488$.

Miall RC, Christensen LO, Cain O, Stanley J (2007) Disruption of state estimation in the human lateral cerebellum. PLoS Biol 5:e316.

Middleton FA, Strick PL (2000) Basal ganglia and cerebellar loops: motor and cognitive circuits. Brain Res Brain Res Rev 31:236-250.

Morton SM, Bastian AJ (2006) Cerebellar contributions to locomotor adaptations during splitbelt treadmill walking. J Neurosci 26:9107-9116.

Nitsche MA, Paulus W (2000) Excitability changes induced in the human motor cortex by weak transcranial direct current stimulation. J Physiol 527:633-639.

Nitsche MA, Paulus W (2001) Sustained excitability elevations induced by transcranial DC motor cortex stimulation in humans. Neurology 57:1899-1901.

Nitsche MA, Liebetanz D, Antal A, Lang N, Tergau F, Paulus W (2003a) Modulation of cortical excitability by weak direct current stimulationtechnical, safety and functional aspects. Suppl Clin Neurophysiol 56:255-276.

Nitsche MA, Fricke K, Henschke U, Schlitterlau A, Liebetanz D, Lang N, Henning S, Tergau F, Paulus W (2003b) Pharmacological modulation of cortical excitability shifts induced by transcranial direct current stimulation in humans. J Physiol 553:293-301.

Oliveri M, Koch G, Torriero S, Caltagirone C (2005) Increased facilitation of the primary motor cortex following $1 \mathrm{~Hz}$ repetitive transcranial magnetic stimulation of the contralateral cerebellum in normal humans. Neurosci Lett 376:188-193.

Pinto AD, Chen R (2001) Suppression of the motor cortex by magnetic stimulation of the cerebellum. Exp Brain Res 140:505-510.

Purpura DP, McMurtry JG (1965) Intracellular activities and evoked potential changes during polarization of motor cortex. J Neurophysiol $28: 166-185$.

Reis J, Swayne OB, Vandermeeren Y, Camus M, Dimyan MA, Harris-Love M, Perez MA, Ragert P, Rothwell JC, Cohen LG (2008) Contribution of transcranial magnetic stimulation to the understanding of cortical mechanisms involved in motor control. J Physiol 586:325-351.

Rossini PM, Barker AT, Berardelli A, Caramia MD, Caruso G, Cracco RQ, Dimitrijevic MR, Hallett M, Katayama Y, Lücking $\mathrm{CH}$, Maertens de Noordhout AL, Marsden CD, Murray NMF, Rothwell JC, Swash M, Tomberg C (1994) Non-invasive electrical and magnetic stimulation of the brain, spinal cord and roots: basic principles and procedures for routine clinical application. Report of an IFCN committee. Electroencephalogr Clin Neurophysiol 91:79-92.

Shepherd GM (2004) The synaptic organization of the brain. New York: Oxford UP.

Stefan K, Cohen LG, Duque J, Mazzocchio R, Celnik P, Sawaki L, Ungerleider L, Classen J (2005) Formation of a motor memory by action observation. J Neurosci 25:9339-9346.

Theoret H, Haque J, Pascual-Leone A (2001) Increased variability of paced finger tapping accuracy following repetitive magnetic stimulation of the cerebellum in humans. Neurosci Lett 306:29-32.

Ugawa Y, Day BL, Rothwell JC, Thompson PD, Merton PA, Marsden CD (1991) Modulation of motor cortical excitability by electrical stimulation over the cerebellum in man. J Physiol 441:57-72.

Ugawa Y, Uesaka Y, Terao Y, Hanajima R, Kanazawa I (1994) Magnetic stimulation of corticospinal pathways at the foramen magnum level in humans. Ann Neurol 36:618-624.

Ugawa Y, Uesaka Y, Terao Y, Hanajima R, Kanazawa I (1995) Magnetic stimulation over the cerebellum in humans. Ann Neurol 37:703-713.

Werhahn KJ, Taylor J, Ridding M, Meyer BU, Rothwell JC (1996) Effect of transcranial magnetic stimulation over the cerebellum on the excitability of human motor cortex. Electroencephalogr Clin Neurophysiol 101:58-66.

Ziemann U, Ishii K, Borgheresi A, Yaseen Z, Battaglia F, Hallett M, Cincotta M, Wassermann EM (1999) Dissociation of the pathways mediating ipsilateral and contralateral motor-evoked potentials in human hand and arm muscles. J Physiol 518:895-906. 